\title{
The association of thyroid function and the risk of kidney function decline: a population-based cohort study
}

\author{
Layal Chaker1,2,3,*, Sanaz Sedaghat 3,*, Ewout J Hoorn², Wendy P J Den Elzen4, \\ Jacobijn Gussekloo5, Albert Hofman ${ }^{3,6}$, M Arfan Ikram ${ }^{3,7}$, Oscar H Franco3, \\ Abbas Dehghan ${ }^{3,+}$ and Robin P Peeters ${ }^{1,2,3,+}$ \\ ${ }^{1}$ Rotterdam Thyroid Center, ${ }^{2}$ Department of Internal Medicine, ${ }^{3}$ Department of Epidemiology, Erasmus University \\ Medical Center, Rotterdam, The Netherlands, ${ }^{4}$ Department of Clinical Chemistry and Laboratory Medicine and \\ ${ }^{5}$ Department of Public Health and Primary Care, Leiden University Medical Center, Leiden, The Netherlands, \\ ${ }^{6}$ Harvard T H Chan School of Public Health, Boston, Massachusetts, USA, and ${ }^{7}$ Department of Neurology, \\ Erasmus University Medical Center, Rotterdam, The Netherlands \\ *(L Chaker and S Sedaghat contributed equally to this work) \\ ${ }^{\dagger}$ (A Dehghan and R P Peeters contributed equally to this work)
}

Correspondence should be addressed to R P Peeters Email r.peeters@erasmusmc.n

\begin{abstract}
Objectives: Thyroid dysfunction has been associated with kidney function decline, but mainly in cross-sectional studies. Therefore, we aimed to determine the association between thyroid and kidney function in a prospective populationbased cohort study longitudinally.

Design: Prospective cohort study.

Methods: Participants aged $\geq 45$ years from the Rotterdam Study with thyroid and kidney function assessment were included. Kidney function and new onset chronic kidney disease (CKD) were defined using estimated glomerular filtration ate (eGFR), with CKD defined as eGFR $<60 \mathrm{~mL} / \mathrm{min} / 1.73 \mathrm{~m}^{2}$ according to the CKD-EPI formula.

Results: We included 5103 participants (mean age of 63.6 years) with a mean follow-up of 8.1 years. Cross-sectionally, higher TSH levels were associated with lower eGFR (Beta $(\beta):-1.75 \mathrm{~mL} / \mathrm{min} ; 95 \%$ confidence interval $(\mathrm{Cl}):-2.17,-1.33)$, in multivariable models adjusting for several cardiovascular risk factors including smoking, hypertension and history of coronary heart disease among others. In contrast, longitudinally, higher TSH levels were associated with less annual eGFR decline ( $\beta$ : $-0.06 \mathrm{~mL} / \mathrm{min} ; \mathrm{Cl}:-0.11,-0.01)$ and lower CKD incidence (odds ratio $0.85, \mathrm{Cl} ; 0.75,0.96$ ). Compared with euthyroid participants, subclinical hyperthyroid individuals had an increased risk for CKD whereas hypothyroid individuals had a decreased risk ( $P$ for trend $=0.04$ ).

Conclusions: Hyperactive thyroid function is associated with increased risk of kidney function decline while hypothyroidism is associated with a decreased CKD risk. More insight is needed in the pathophysiological pathways connecting high thyroid function and kidney function decline.

\section{Introduction}

Thyroid hormone has an impact on renal tubular function, the renin-angiotensin system and is associated with haemodynamic and cardiovascular alterations that interfere with renal blood flow $(1,2,3)$. Hypothyroidism has been associated with a low glomerular filtration rate and chronic kidney disease (CKD) $(4,5)$. On the
() 2016 European Society of Endocrinology Printed in Great Britain other hand, CKD can also lead to changes in thyroid function, for example through non-thyroidal illness, metabolic acidosis or selenium deficiency $(6,7,8)$. The co-existence of (subclinical) hypothyroidism and renal dysfunction in patients has prompted investigation into the effects of thyroid hormone replacement therapy

Published by Bioscientifica Ltd. 
on CKD $(9,10)$. However, most evidence concerning the association between thyroid and kidney function has been based on cross-sectional studies and included mostly patients with CKD or thyroid dysfunction $(4,11,12)$. The understanding of the temporal relationship between thyroid and kidney dysfunction therefore remains largely unexplored. So far, only two prospective studies in specific populations have investigated this association with conflicting results $(5,13)$. One study was conducted in 555 participants of 85 years old and found no association between thyroid status and change in renal function (13). The second study was a large cohort study including only euthyroid subjects and reported an increased CKD risk with low-normal thyroid function (5). To date, there are no large population-based cohort studies investigating the continuous and full range of thyroid function and the risk of kidney function decline and CKD. Therefore, in the current prospective study, we aimed to investigate the association of thyroid function with the risk of kidney function decline and CKD in the general population.

\section{Subjects and methods}

\section{Study population}

The Rotterdam Study (RS) is a prospective populationbased cohort study that investigates determinants and occurrence of cardiovascular, neurological, ophthalmologic, psychiatric and endocrine diseases in the middle-aged and elderly living in Ommoord, a suburb of Rotterdam. The aims and design of the RS have been described in detail elsewhere (14). For this analysis, we included participants from three independent cohorts within the RS. The RS Cohort 1 (RSI) started in 1990 and included a total of 7983 participants (response rate 78 percent) aged 55 years and older. RS Cohort II (RSII) includes a total of 3011 participants (response rate 67 percent) aged 55 years and older and baseline data were collected from 2000 to 2001. For the RS Cohort 3 (RSIII), all residents of Ommoord aged 45 years and over and who had not been invited before, were asked to participate and baseline data were collected from 2006 to 2008. A total of 3932 participants entered the study (response rate 65 percent). Participants from study cohorts RSI (RSI-3), RSII (RSII-1) and RSIII (RSIII-1) were eligible for the study if TSH and FT4 measurements as well as serum creatinine were available at baseline and follow-up.

The Medical Ethics Committee of the Erasmus University approved the study protocols and by the Ministry of Health, Welfare and Sport of the Netherlands, implementing the 'Wet Bevolkingsonderzoek: ERGO (Population Studies Act: Rotterdam Study)'. All included participants provided a written informed consent in accordance with the Declaration of Helsinki to participate in the study and to obtain information from their family physicians.

\section{Assessment of thyroid function}

We performed thyroid function tests measurements in the three RS cohorts in 9762 subjects. All samples were collected between 1997 and 2008, depending on the cohort, and measured using the same methods and assay. Thyroid function assessment was performed for TSH and FT4 in all three cohorts in serum samples stored at $-80^{\circ} \mathrm{C}$ (the electrochemiluminescence immunoassay for thyroxine, thyrotropin and thyroid perioxidase antibodies, 'ECLIA', Roche). We determined the cut-off values for normal range of TSH as $0.4-4.0 \mathrm{IU} / \mathrm{L}$ and FT4 as $11-25 \mathrm{pmol} / \mathrm{L}$ (0.86-1.94 ng/dL) according to national guidelines as well as our previous studies. Thyroid function was defined as euthyroid if TSH was in the normal range $(0.4-4.0 \mathrm{IU} / \mathrm{L})$. Overt hyperthyroidism was defined by a TSH level below the normal range $(<0.4 \mathrm{IU} / \mathrm{L})$ and FT4 above the normal range ( $>25 \mathrm{pmol} / \mathrm{L})$, while in subclinical hyperthyroidism FT4 values were within the normal range (11-25 pmol/L). Overt hypothyroidism was defined by TSH levels above the upper range of normal (>4.0IU/L) and FT4 below the normal range $(<11 \mathrm{pmol} / \mathrm{L})$, while in subclinical hypothyroidism FT4 values were within the normal range (11-25 pmol/L). The laboratory measurements of thyroid function were not reported back to the participants and any thyroid therapy prescribed by their own GP or specialist is within the context of regular treatment and blinded to measurements of the RS.

\section{Kidney function and CKD}

Serum creatinine was determined using an enzymatic assay method using the same technique at the same laboratory for all three cohorts. Inter- and intraassay coefficient variations were $<0.92 \%$ and $<1.37 \%$, respectively (15). Creatinine values were standardized to isotope-dilution mass spectrometry (IDMS)-traceable measurements. In order to calibrate, we aligned the mean values of serum creatinine with serum creatinine values of the participants of the Third National Health and Nutrition Examination Survey (NHANES III) in different 
Table 1 Baseline characteristics of the study population. Categorical variables are presented as numbers (percentages), continuous variables as means (standard deviations) and TSH is presented as median (interquartile range).

\begin{tabular}{l} 
Characteristics \\
\hline$n$ \\
Age (years) \\
Men \\
Systolic blood pressure $(\mathrm{mmHg})$ \\
Diastolic blood pressure $(\mathrm{mmHg})$ \\
Smoking \\
$\quad$ Current \\
Former \\
Total cholesterol (mmol/L) \\
HDL cholesterol (mmol/L) \\
Body mass index (kg/m²) \\
Diabetes mellitus \\
History of coronary heart disease \\
Glomerular filtration rate (creatinine) \\
$\quad$ (mL/min/1.73 $\mathrm{m}^{2}$ ) \\
TSH (IU/L) \\
FT4 (pmol/L) \\
Levothyroxine use
\end{tabular}

\begin{tabular}{c}
\hline Values \\
\hline 5103 \\
$63.6(9.4)$ \\
$2227(43.6)$ \\
$136.3(19.8)$ \\
$80.3(11.0)$ \\
\\
$1057(20.7)$ \\
$2419(47.4)$ \\
$5.7(1.01)$ \\
$1.4(0.4)$ \\
$27.3(4.2)$ \\
$409(8.0)$ \\
$188(3.7)$ \\
$84.1(14.1)$
\end{tabular}

$2.0(1.4,2.8)$

$15.6(2.2)$

$150(2.9)$
The following variables had missing data: blood pressure $(n=10)$, smoking ( $n=19), \mathrm{HDL}$ : high-density lipoprotein cholesterol $(n=14)$, body mass index $(n=21)$, diabetes $(n=40)$ and coronary heart disease $(n=30)$.

gender and age groups $(<50,50-59,60-69, \geq 70$ years). Estimated glomerular filtration rate (eGFR) was calculated according to the CKD Epidemiology Collaboration (CKD-EPI) formula (16). To calculate the annual eGFR decline, we first subtracted the eGFR estimates of the follow-up examination from the eGFR estimates at baseline and then divided by the time, in years, between the two visits. For these analyses we used all participants with available eGFR measurements. CKD was defined as eGFR $<60 \mathrm{~mL} / \mathrm{min} / 1.73 \mathrm{~m}^{2}$. New CKD cases were defined among the individuals free of CKD at baseline (defined by eGFR $>60 \mathrm{~mL} / \mathrm{min} / 1.73 \mathrm{~m}^{2}$ ), who had a decline in eGFR to less than $60 \mathrm{~mL} / \mathrm{min} / 1.73 \mathrm{~m}^{2}$ between the two periodical examinations.

\section{Baseline measurements}

Information on smoking was derived from computerized baseline questionnaires and categorized in current, previous and never smokers. Systolic and diastolic blood pressure were calculated as the average of two consecutive measurements, using a randomzero mercury sphygmomanometers. Information on antihypertensive medication use was based on home interview at baseline. Serum glucose, total serum cholesterol and high-density lipoprotein cholesterol
(HDL-C) were measured using standard laboratory techniques. History of diabetes was defined by a repeated impaired fasting glucose $\geq 7$ or use of antiglycaemic medication at baseline. Body mass index (BMI) was calculated as weight in kilograms divided by height squared in meters.

\section{Statistical analysis}

We first jointly analysed RS cohorts I and II and then replicated the results in RS cohort III, which provided similar sample sizes for discovery and replication. We present the combined multivariable analyses (RSI, RSII and RSIII) as our primary results. Linear regression models were used to evaluate the association of FT4 or TSH with baseline eGFR (based on creatinine) or decline in eGFR. Logistic regression models were performed to estimate the odds ratio (OR) for the association of FT4 or TSH with incident CKD.

Due to a skewed distribution, TSH was log-transformed for all continuous statistical analyses.

Our primary model included adjustment for age and sex. The multivariable models were additionally adjusted for systolic blood pressure, diastolic blood pressure, smoking, total cholesterol, high-density lipoprotein cholesterol, diabetes mellitus, antihypertensive medications and BMI.

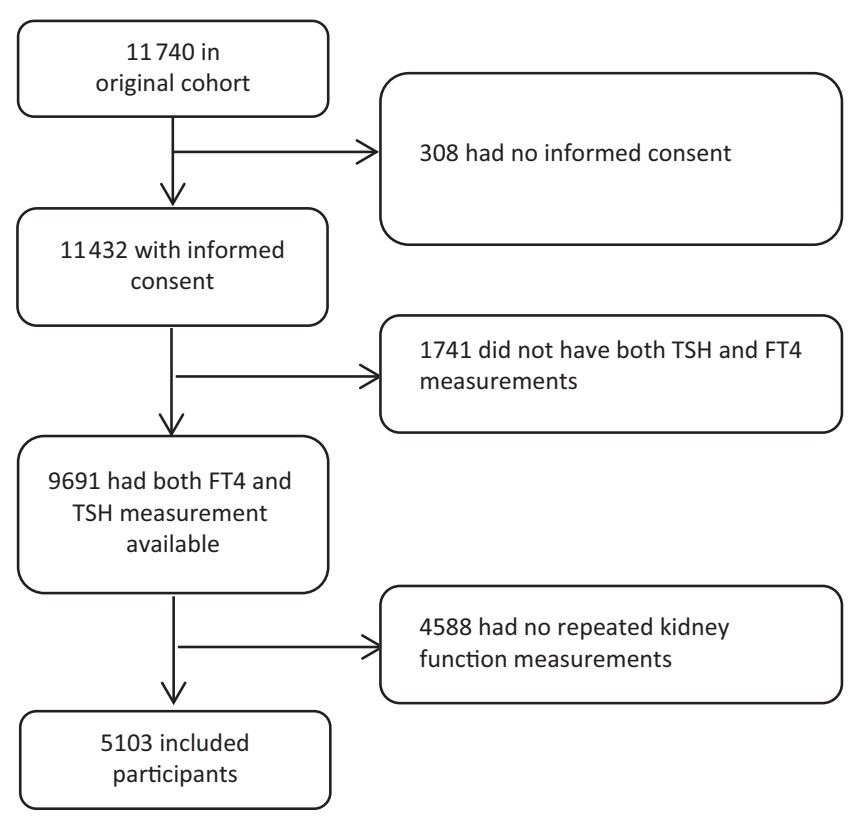

Figure 1

Flow chart of participants' inclusion. 


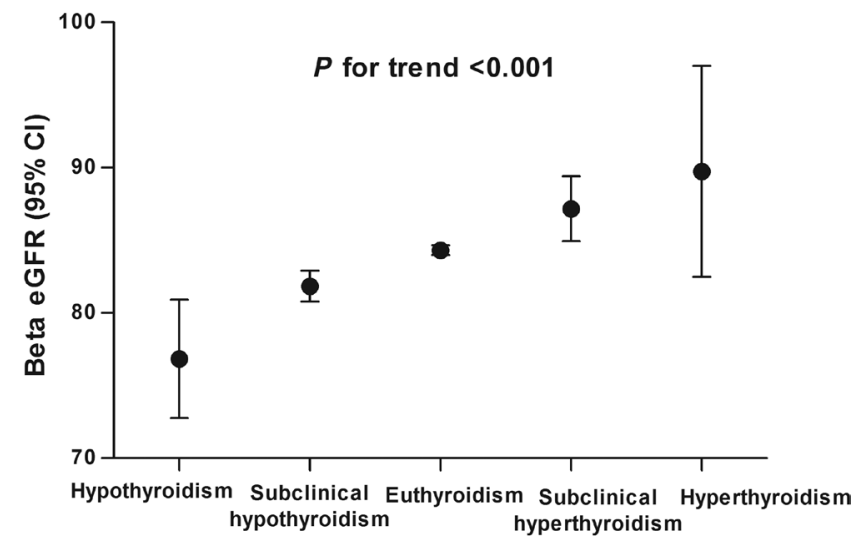

\section{Figure 2}

Mean values of eGFR in categories of thyroid function. From 5013 participants, 4488 were euthyroid, 467 subclinical hypothyroid, 32 hypothyroid, 106 subclinical hyperthyroid and 10 were hyperthyroid. These analyses were adjusted for age, sex, systolic blood pressure, diastolic blood pressure, antihypertensive medication, smoking, total cholesterol, high-density lipoprotein cholesterol, diabetes mellitus, history of coronary heart disease and body mass index.

We performed an analysis of covariance where mean values of decline in eGFR were compared across categories of thyroid function. We also assessed incidence of CKD in categories of thyroid function using logistic regression. For the thyroid function analyses all thyroid hormone users were excluded.

We performed multiple imputation for missing data in the covariates ( $<1 \%$ for all covariates), using a Markov chain Monte Carlo method.

We assessed differential risk by age or sex by adding an interaction term with the exposure variable. For the longitudinal TSH and FT4 analyses we performed the following sensitivity analyses: (1) restricting the analyses to participants with TSH and FT4 values within the normal range and (2) excluding participants using thyroid function altering medication (including levothyroxine, anti-thyroid drugs, amiodarone and corticosteroids) or prevalent thyroid disease. There was no departure from linearity for the TSH, FT4 or thyroid function analyses. All statistical analyses were performed using SPSS version 21 (SPSS IBM). Reporting of this study is according to the STROBE Statement.

\section{Results}

Baseline characteristics of included participants from the Rotterdam Study (14), a population-based prospective cohort study, are shown in Table 1. Out of the 5103 included participants (Fig. 1) who had thyroid function and serum creatinine measurements, $43.6 \%$ were male (mean age of 64 years), with a mean follow-up of 8.1 years. A total of 4488 participants were euthyroid (87.9\%), 467 had subclinical hypothyroidism (9.2\%), 32 had hypothyroidism $(0.6 \%), 106$ were subclinically hyperthyroid $(2.1 \%)$ and 10 were hyperthyroid $(0.2 \%)$ at baseline.

\section{Cross-sectional analyses}

Compared with euthyroid subjects, hyperthyroid participants had a higher eGFR, while participants with hypothyroidism had a lower eGFR at baseline (Fig. 2). There was a significant $P$ for trend across the different thyroid function categories for eGFR at baseline $(P<0.001)$. Higher TSH levels were associated

Table 2 Cross-sectional association of thyroid function markers with kidney function. Data are presented as $\beta(95 \% \mathrm{Cl})$.

\begin{tabular}{|c|c|c|c|}
\hline & \multicolumn{3}{|c|}{ eGFR } \\
\hline & RS I and II & RS III & Combined \\
\hline$n$ & 2524 & 2579 & 5103 \\
\hline \multicolumn{4}{|l|}{ TSH } \\
\hline Model I & $-2.10(-2.64,-1.56)$ & $-1.48(-2.14,-0.81)$ & $-1.83(-2.25,-1.41)$ \\
\hline Model II & $-2.00(-2.53,-1.46)$ & $-1.40(-2.06,-0.73)$ & $-1.75(-2.17,-1.33)$ \\
\hline \multicolumn{4}{|l|}{ FT4 } \\
\hline Model I & $0.02(-0.18,0.22)$ & $-0.05(-0.27,0.17)$ & $-0.02(-0.17,0.13)$ \\
\hline Model II & $-0.01(-0.21,0.19)$ & $-0.06(-0.28,0.16)$ & $-0.04(-0.19,0.10)$ \\
\hline
\end{tabular}

Model I: Adjusted for age, sex and cohort effect.

Model II: Additionally adjusted for systolic blood pressure, diastolic blood pressure, smoking, total cholesterol, high-density lipoprotein cholesterol, diabetes mellitus, antihypertensive medications, history of coronary heart disease, and body mass index.

$\mathrm{Cl}$, confidence interval; eGFR, estimated glomerular filtration rate; FT4, free thyroxine; TSH, thyroid-stimulating hormone; RS: Rotterdam Study. 

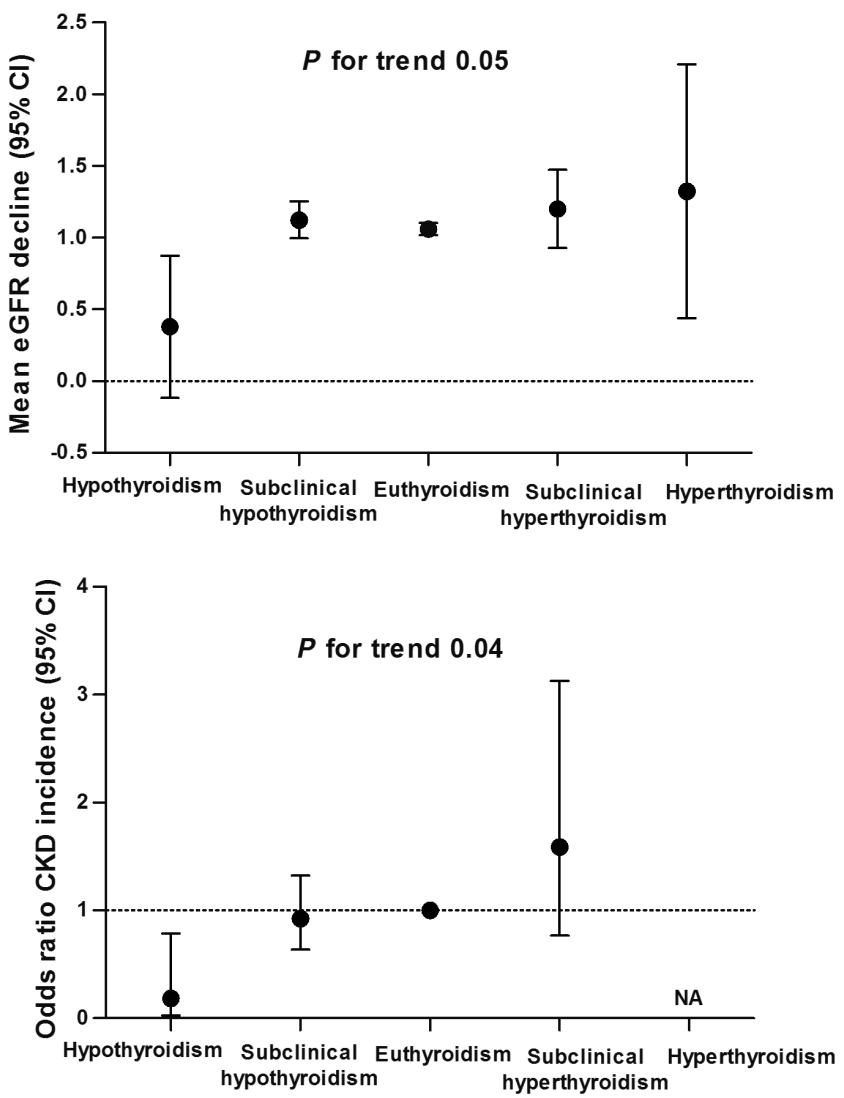

Figure 3

Mean values of eGFR decline and odds ratios of CKD incident in categories of thyroid function. From 5103 participants 4488 were euthyroid, 467 subclinical hypothyroid, 32 hypothyroid, 106 subclinical hyperthyroid and 10 were hyperthyroid. These analyses were adjusted for age, sex, systolic blood pressure, diastolic blood pressure, antihypertensive medication, smoking, total cholesterol, high-density lipoprotein cholesterol, diabetes mellitus, history of coronary heart disease and body mass index. NA, not available, due to small number of events in this strata.

CKD (OR: 0.85; 95\% CI: 0.75, 0.96, Table 3). There was no association between TSH in the normal range of thyroid function and decline in eGFR or incident CKD (Table 4). When excluding subjects with clinically known thyroid disease or thyroid medication use (Table 4), the estimates were similar, but lost significance. Overall, FT4 values were not associated with eGFR decline or incident CKD (Table 3$)$. There was no differential risk by age or sex ( $P$ for interaction $>0.15$ ). Additionally adjusting for BMI change over time did not change risk estimates (data not shown).

Participants with subclinical hyperthyroidism had more eGFR decline and hypothyroid participants had less decline in eGFR (Fig. 3). For the CKD analyses, the hyperthyroidism category was excluded due to small number of events and therefore infinite estimates. Participants with hypothyroidism had a decreased risk of incident CKD, whereas participants with subclinical hyperthyroidism had an increased risk of $\mathrm{CKD}$. The $P$ for trend was a significant across the different categories of thyroid function for incident CKD $(P=0.04)$ and for mean eGFR decline $(P=0.05)$.

\section{Discussion}

To our knowledge, this is the first prospective population-based study to report an association between high thyroid function and kidney function decline in the general population. We report an increased risk of CKD and eGFR decline in subjects with lower TSH levels or hyperthyroid state, while hypothyroidism seems protective. This is irrespective of age, sex and several cardiovascular risk factors including blood pressure, smoking and cholesterol.

A large study in over 460000 veterans reports an inverse association between eGFR and the risk of hypothyroidism (17). However, this study is not comparable with ours as it only includes participants with CKD defined as an eGFR of $<60 \mathrm{~mL} / \mathrm{min} / 1.73 \mathrm{~m}^{2}$, while our population reflects the general population. Only two previous studies have longitudinally investigated the association between thyroid function and kidney function. However, these studies focused on specific study populations with small sample size, investigated specific thyroid states instead of the continuous range of thyroid function and their results were conflicting. While Zhang et al. (5) found a modestly increased risk of CKD with high-normal TSH levels in $>100000$ euthyroid subjects, Meuwese et al. (13) found no relation between thyroid function and kidney function in 555 subjects of 85 years old in the longitudinal analyses. The differences between our data and these studies may be explained by various factors.

The Kangbuk Samsung Health (KSH) Study looked specifically in euthyroid subjects, whereas our results concern the continuous and full spectrum of thyroid function (5). When we restrict our analyses to subjects with thyroid function within the reference range, we find no association of thyroid function with kidney function decline or CKD. The CKD-EPI equation, which was used in $\mathrm{KSH}$, has shown to introduce inaccuracy among certain racial-ethnic groups such as Asians (18). In addition, the mean age of the populations and follow-up times differ, since our population is substantially older (mean age of 38 vs 63.6 years), and had a longer follow-up 
(3.5 vs 8.2 years of follow-up). Finally, selection bias cannot be excluded from the KSH Study due to its design: screening health service of employees. The Leiden 85-plus Study, which focused on older adults, included a relatively small number of participants $(n=555)$ and does not report information on CKD at baseline and during follow-up. The mean annual change presented for hyperthyroidism (eGFR $-1 \mathrm{~mL} / \mathrm{min} / 1.73 \mathrm{~m}^{2}$ ) and hypothyroidism (eGFR $+0.5 \mathrm{~mL} / \mathrm{min} / 1.73 \mathrm{~m}^{2}$ ) compared with euthyroidism are in line with our results, but did not reach statistical significance in that study, possibly due to the lower numbers. Furthermore, in this study we also conducted analyses with TSH as a continuous variable, where again we observed a protective effect of higher TSH values (i.e. lower thyroid function).

Most, predominantly cross-sectional, studies to date have focused on the hypothesis that hypothyroidism gives rise to several cardiovascular risk factors and circulatory changes through which kidney function could be affected $(5,13,19,20)$. In our study we confirm the cross-sectional association between (subclinical) hypothyroidism and lower eGFR. However, cross-sectional studies cannot provide information on the temporal relationship between thyroid function and kidney function and are prone to reverse causation.

Therefore, the major aim of this study was to address the issue of temporality by conducting longitudinal analyses. In our study, participants with hypothyroidism had a decreased risk of incident CKD and less eGFR decline while participants with subclinical hyperthyroidism had an increased risk of CKD and more eGFR decline. No studies to date had focused on the possible association between a high thyroid function state and renal dysfunction even though both low and high state of thyroid function are associated with cardiovascular risk factors and disease. Effects of subclinical hyperthyroidism include atrial fibrillation, increase in left ventricular mass index, reduced exercise tolerance, reduced heart rate variability and an increase in markers of coagulation $(21,22,23)$. Furthermore, thyroid hormone is known to activate the renin-angiotensin system (2), which may cause efferent arteriolar vasoconstriction and lead to glomerular hypertension and hyperfiltration (24). The opposite relationship on eGFR in our cross-sectional and longitudinal analysis is similar to what has been described for diabetic nephropathy, which is also characterized by a higher eGFR initially (due to hyperfiltration), but by eGFR loss and CKD in the long run (25).

The seemingly discrepant findings between crosssectional and prospective analyses on thyroid function and kidney decline could be explained by distinctly different mechanisms. With cross-sectional data we may detect the bidirectional association of kidney and thyroid function, whereas with longitudinal data we could measure the deleterious effect of long-term exposure to high thyroid function state on kidney function. More research is needed to explore the difference between these two mechanisms to further characterize the longterm effects of variations of thyroid function on kidney function and disease. This is especially important when considering thyroid hormone replacement therapy in subclinical hypothyroidism.

Strengths of our study include the large number of participants, detailed information on a wide variety of confounders and long follow-up period. Study limitations include the relatively small number of participants with hyperthyroidism and measuring thyroid function only once at baseline. In addition, information regarding the aetiology of CKD cases is not present in the Rotterdam Study. Albuminuria is a strong predictor of kidney function decline, but unfortunately was not available in our cohort at baseline; adjusting our results for albumin-creatinine ratio at follow-up in a subset of participants did not change our findings (data not shown). In addition, we were limited by the number of eGFR measurements and were only able to use two measurements to define a slope. Furthermore, the Rotterdam Study consisted mainly of a white population of 45 years and older and might therefore not be generalizable to other populations. Finally, because we primarily used creatinine-based estimating equations for GFR, we cannot exclude effects of thyroid hormone on the muscle metabolism of creatinine or its tubular secretion.

In conclusion, high thyroid function is associated with an increased risk of incident CKD while hypothyroidism is associated with a decreased CKD risk. Further research should focus on the association between high thyroid function and kidney function and possible pathophysiological mechanisms.

\section{Declaration of interest}

The authors declare that there is no conflict of interest that could be perceived as prejudicing the impartiality of the research reported.

\section{Funding}

Prof R P Peeters and Dr Chaker are supported by a Zon-MWTOP grant (nr 91212044) and an Erasmus MCMRACE grant. Prof Dr R P Peeters has received lecture and consultancy fees from Genzyme. Prof O H Franco works in ErasmusAGE, a centre for ageing research across the life course funded by Nestlé Nutrition (Nestec Ltd.), Metagenics Inc., and AXA. Nestlé Nutrition (Nestec Ltd.), Metagenics Inc. and AXA had no role in the design and conduct of the study; collection, management, analysis and interpretation of the data; and preparation, review or approval of the manuscript. 
The Rotterdam Study is supported by the Erasmus MC and Erasmus University Rotterdam; the Netherlands Organization for Scientific Research (NWO); the Netherlands Organization for Health Research and Development (ZonMw); the Research Institute for Diseases in the Elderly (RIDE); the Netherlands Genomics Initiative; the Ministry of Education, Culture and Science; the Ministry of Health Welfare and Sports; the European Commission (DG XII); and the Municipality of Rotterdam. The funding sources had no involvement in the collection, analysis, writing, interpretation or in the decision to submit the paper for publication.

\section{Author contribution statement}

A $\mathrm{H}, \mathrm{A} \mathrm{D}, \mathrm{OHF}, \mathrm{M} \mathrm{A} \mathrm{I}$ and R P P designed the study, provided funding and supervision. S S, L C and E J H collected analysed the data. S S, L C, E J H, W P J D E, J G, A D and R P P interpreted the results and drafted the manuscript. All authors critically revised the manuscript for important intellectual content

\section{Acknowledgements}

We are grateful to the study participants, the staff from the Rotterdam Study, and participating general practitioners and pharmacists.

\section{References}

1 Meuwese CL \& Carrero JJ. Chronic kidney disease and hypothalamicpituitary axis dysfunction: the chicken or the egg? Archives of Medical Research 201344 591-600. (doi:10.1016/j.arcmed.2013.10.009)

2 Vargas F, Rodriguez-Gomez I, Vargas-Tendero P, Jimenez E \& Montiel M. The renin-angiotensin system in thyroid disorders and its role in cardiovascular and renal manifestations. Journal of Endocrinology 2012213 25-36. (doi:10.1530/JOE-11-0349)

3 Iglesias P \& Diez JJ. Thyroid dysfunction and kidney disease. European Journal of Endocrinology 2009160 503-515. (doi:10.1530/EJE-08-0837)

4 Asvold BO, Bjoro T \& Vatten LJ. Association of thyroid function with estimated glomerular filtration rate in a population-based study: the HUNT study. European Journal of Endocrinology 2011164 101-105. (doi:10.1530/EJE-10-0705)

5 Zhang Y, Chang Y, Ryu S, Cho J, Lee WY, Rhee EJ, Kwon MJ, Pastor-Barriuso R, Rampal S, Han WK et al. Thyroid hormone levels and incident chronic kidney disease in euthyroid individuals: the Kangbuk Samsung Health Study. International Journal of Epidemiology 201443 1624-1632. (doi:10.1093/ije/dyu126)

6 Rhee CM, Brent GA, Kovesdy CP, Soldin OP, Nguyen D, Budoff MJ, Brunelli SM \& Kalantar-Zadeh K. Thyroid functional disease: an under-recognized cardiovascular risk factor in kidney disease patients. Nephrology Dialysis Transplantation 201530 724-737. (doi:10.1093/ ndt/gfu024)

7 Schomburg L. Selenium, selenoproteins and the thyroid gland: interactions in health and disease. Nature Reviews Endocrinology 2012 8 160-171. (doi:10.1038/nrendo.2011.174)

8 Wiederkehr MR, Kalogiros J \& Krapf R. Correction of metabolic acidosis improves thyroid and growth hormone axes in haemodialysis patients. Nephrology Dialysis Transplantation 200419 1190-1197. (doi:10.1093/ndt/gfh096)

9 Shin DH, Lee MJ, Kim SJ, Oh HJ, Kim HR, Han JH, Koo HM, Doh FM, Park JT, Han SH et al. Preservation of renal function by thyroid hormone replacement therapy in chronic kidney disease patients with subclinical hypothyroidism. Journal of Clinical Endocrinology and Metabolism 201297 2732-2740. (doi:10.1210/jc.2012-1663)
10 Shin DH, Lee MJ, Lee HS, Oh HJ, Ko KI, Kim CH, Doh FM, Koo HM, Kim HR, Han JH et al. Thyroid hormone replacement therapy attenuates the decline of renal function in chronic kidney disease patients with subclinical hypothyroidism. Thyroid 201323 654-661. (doi:10.1089/thy.2012.0475)

11 Karanikas G, Schutz M, Szabo M, Becherer A, Wiesner K, Dudczak R $\&$ Kletter K. Isotopic renal function studies in severe hypothyroidism and after thyroid hormone replacement therapy. American Journal of Nephrology 200424 41-45. (doi:10.1159/000075628)

12 Woodward A, McCann S \& Al-Jubouri M. The relationship between estimated glomerular filtration rate and thyroid function: an observational study. Annals of Clinical Biochemistry 200845 515-517. (doi:10.1258/acb.2008.007248)

13 Meuwese CL, Gussekloo J, de Craen AJ, Dekker FW \& den Elzen WP. Thyroid status and renal function in older persons in the general population. Journal of Clinical Endocrinology and Metabolism 201499 2689-2696. (doi:10.1210/jc.2013-3778)

14 Hofman A, Brusselle GG, Darwish Murad S, van Duijn CM, Franco OH, Goedegebure A, Ikram MA, Klaver CC, Nijsten TE, Peeters RP et al. The Rotterdam Study: 2016 objectives and design update. European Journal of Epidemiology 201530 661-708. (doi:10.1007/s10654-015-0082-x)

15 Sedaghat S, Cremers LG, de Groot M, Hoorn EJ, Hofman A, van der Lugt A, Franco OH, Vernooij MW, Dehghan A \& Ikram MA. Kidney function and microstructural integrity of brain white matter. Neurology 201585 154-161. (doi:10.1212/WNL. $0000000000001741)$

16 Inker LA, Schmid CH, Tighiouart H, Eckfeldt JH, Feldman HI, Greene T, Kusek JW, Manzi J, Van Lente F, Zhang YL et al. Estimating glomerular filtration rate from serum creatinine and cystatin C. New England Journal of Medicine 2012367 20-29. (doi:10.1056/NEJMoa1114248)

17 Rhee CM, Kalantar-Zadeh K, Streja E, Carrero JJ, Ma JZ, Lu JL \& Kovesdy CP. The relationship between thyroid function and estimated glomerular filtration rate in patients with chronic kidney disease. Nephrology Dialysis Transplantation 201530 282-287. (doi:10.1093/ ndt/gfu303)

18 Pottel H, Hoste L, Delanaye P, Cavalier E \& Martens F. Demystifying ethnic/sex differences in kidney function: is the difference in (estimating) glomerular filtration rate or in serum creatinine concentration? Clinica Chimica Acta 2012413 1612-1617. (doi:10.1016/j.cca.2012.04.034)

19 Kreisman SH \& Hennessey JV. Consistent reversible elevations of serum creatinine levels in severe hypothyroidism. Archives of Internal Medicine 1999159 79-82. (doi:10.1001/archinte.159.1.79)

20 Lo JC, Chertow GM, Go AS \& Hsu CY. Increased prevalence of subclinical and clinical hypothyroidism in persons with chronic kidney disease. Kidney International 200567 1047-1052. (doi:10.1111/ j.1523-1755.2005.00169.x)

21 Biondi B \& Cooper DS. The clinical significance of subclinical thyroid dysfunction. Endocrine Reviews 200829 76-131. (doi:10.1210/er.2006-0043)

22 Cooper DS \& Biondi B. Subclinical thyroid disease. Lancet 2012379 1142-1154. (doi:10.1016/S0140-6736(11)60276-6)

23 Yavuz DG, Yazici D, Toprak A, Deyneli O, Aydin H, Yuksel M \& Akalin S. Exogenous subclinical hyperthyroidism impairs endothelial function in nodular goiter patients. Thyroid $2008 \mathbf{1 8} 395-400$. (doi:10.1089/thy.2007.0299)

24 Helal I, Fick-Brosnahan GM, Reed-Gitomer B \& Schrier RW. Glomerular hyperfiltration: definitions, mechanisms and clinical implications. Nature Reviews Nephrology 20128 293-300. (doi:10.1038/nrneph.2012.19)

25 Pugliese G. Updating the natural history of diabetic nephropathy. Acta Diabetologica 201451 905-915. (doi:10.1007/s00592-014-0650-7)

Received 23 June 2016

Revised version received 2 September 2016

Accepted 29 September 2016 